\title{
MinFinder: Locating all the local minima of a function ${ }^{\sqrt{2}}$
}

\author{
Ioannis G. Tsoulos ${ }^{a}$, Isaac E. Lagaris ${ }^{b, *, 1}$ \\ a Department of Computer Science, University of Ioannina, P.O. Box 1186, Ioannina 45110 Greece \\ b Physics Department, University of South Africa, P.O. Box 392, 0001 Pretoria, South Africa
}

Received 19 July 2005; received in revised form 22 September 2005; accepted 7 October 2005

Available online 14 November 2005

\begin{abstract}
A new stochastic clustering algorithm is introduced that aims to locate all the local minima of a multidimensional continuous and differentiable function inside a bounded domain. The accompanying software (MinFinder) is written in ANSI C++. However, the user may code his objective function either in $\mathrm{C}++, \mathrm{C}$ or Fortran 77. We compare the performance of this new method to the performance of Multistart and Topographical Multilevel Single Linkage Clustering on a set of benchmark problems.
\end{abstract}

\section{Program summary}

Title of program: MinFinder

Catalogue identifier: ADWU

Program summary URL: http://cpc.cs.qub.ac.uk/summaries/ADWU

Program obtainable from: CPC Program Library, Queen's University of Belfast, N. Ireland

Computer for which the program is designed and others on which is has been tested: The tool is designed to be portable in all systems running the GNU C++ compiler

Installation: University of Ioannina, Greece

Programming language used: GNU-C++, GNU-C, GNU Fortran 77

Memory required to execute with typical data: $200 \mathrm{~KB}$

No. of bits in a word: 32

No. of processors used: 1

Has the code been vectorized or parallelized?: no

No. of lines in distributed program, including test data, etc.: 5797

No. of bytes in distributed program, including test data, etc.: 588121

Distribution format: gzipped tar file

Nature of the physical problem: A multitude of problems in science and engineering are often reduced to minimizing a function of many variables. There are instances that a local optimum does not correspond to the desired physical solution and hence the search for a better solution is required. Local optimization techniques can be trapped in any local minimum. Global optimization is then the appropriate tool. For example, solving a non-linear system of equations via optimization, employing a "least squares" type of objective, one may encounter many local minima that do not correspond to solutions, i.e. they are far from zero.

Method of solution: Using a uniform pdf, points are sampled from the rectangular search domain. A clustering technique, based on a typical distance and a gradient criterion, is used to decide from which points a local search should be started. The employed local procedure is a BFGS version due to Powell. Further searching is terminated when all the local minima inside the search domain are thought to be found. This is accomplished via the double-box rule.

\footnotetext{
th This paper and its associated computer program are available via the Computer Physics Communications homepage on ScienceDirect (http://www.sciencedirect. com/science/journal/00104655).

* Corresponding author.

E-mail address: lagaris@cs.uoi.gr (I.E. Lagaris).

1 Permanen address: Department of Computer Science, University of Ioannina, P.O. Box 1186, Ioannina 45110, Greece.
} 
Typical running time: Depending on the objective function

(c) 2005 Elsevier B.V. All rights reserved.

PACS: 02.60.-x; 02.60.Pn; 07.05.Kf; 02.70.Lq

Keywords: Global optimization; Stochastic methods; Monte Carlo; Clustering; Region of attraction

\section{Introduction}

The task of locating all the local minima of a multidimensional continuous differentiable function $f(x): S \subset R^{n} \rightarrow R$ may be defined as:

Find all $x_{i}^{*} \in S \subset R^{n}$ that satisfy:

$$
x_{i}^{*}=\arg \min _{x \in S_{i}} f(x), \quad S_{i}=S \cap\left\{x,\left|x-x_{i}^{*}\right|<\epsilon\right\} .
$$

Here $S$ is a hyper box defined as:

$$
S=\left[a_{1}, b_{1}\right] \otimes\left[a_{2}, b_{2}\right] \otimes \cdots \otimes\left[a_{n}, b_{n}\right] .
$$

This problem appears frequently as a subproblem in a variety of scientific applications. Among the several methods employed to treat this problem, stochastic methods seem to be the most popular, most probably due to both their effectiveness and implementation simplicity. An important subclass of stochastic methods are the so-called clustering techniques, pioneered by Becker and Lago [1], Törn [2], Boender et al. [3], Rinnooy Kan and Timmer [4,5]. Clustering techniques are based on the "multistart" algorithm and their goal is to limit the number of local search applications. A cluster is defined as a set of points that are believed to belong to the region of attraction of the same minimum, and hence only one local search is (optimally) required to locate it. The region of attraction of a local minimum $x^{*}$ is defined as:

$$
A\left(x^{*}\right)=\left\{x: x \in S \subset R^{n}, L(x)=x^{*}\right\},
$$

where $L(x)$ is the point where the local search procedure $L$ terminates when started at point $x$. Here $L$ is supposed to be a deterministic local optimization method such as BFGS [8], Steepest Descent, Modified Newton, etc. The present work is a clustering technique based on the "Topographical Multilevel Single Linkage" (TMLSL) of Ali and Storey [6]. The modifications we present are important and render the technique significantly more efficient.

In Section 2 we present the proposed algorithm and in Section 3 we present the results of numerical experiments, along with our conclusions. In Section 4 we present the documentation of the related software, describing its distribution, installation and use. In Appendix A we list the test functions employed in our experiments for evaluating the performance of the proposed scheme.

\section{Description of the algorithm}

\subsection{Steps of the algorithm}

In the following by $X^{*}$ we denote the set of the local minima collected so far. Initially $X^{*}=\emptyset$. The steps of the proposed algorithm are as:

\section{Checking step:}

- Set $V=\emptyset$.

- Set $T$ as the set of $N$ points sampled from the Double Box procedure.

- Forall $x \in T$ do

- Check if $x$ is a valid starting point (validated by a procedure described later), and if so add it to $V$.

- Endforall

\section{Enrichment step:}

- If $\frac{|V|}{N}<\frac{1}{2}$ then

$-N=\min \left(N+\frac{N}{10}, \mathrm{NMAX}\right)$, where NMAX is a predefined upper limit for the number of samples in each generation. This step prevents the algorithm from performing an insufficient exploration of the search space. The typical value for this parameter is $\mathrm{NMAX}=100$.

- Endif 


\section{Main step:}

- Forall $x \in V$ do

- If $x$ is considered as start point (the validation procedure is once more performed, because a point that was considered as a start point earlier may no longer be due to the presence of new local minima) then

* Start a local search $y=L(x)$.

* Compute the typical distance $r_{t}$ using Eq. (1).

$*$ If $y \notin X^{*}$ then

. Set $X^{*}=X^{*} \cup y$.

* Endif

- Endif

- Endforall

A point $x$ is to be considered as start point if none of the following conditions holds:

- There is an already located minimum $z$ that satisfies the conditions

1. $(x-z)^{\mathrm{T}}(\nabla f(x)-\nabla f(z))>0$.

2. $|x-z|<\min _{i, j, i \neq j}\left|z_{i}-z_{j}\right|, z_{i} \in X^{*}, z_{j} \in X^{*}$.

- $x$ is near to another point $y \in V$ that satisfies the conditions

1. $|x-y|<r_{t}$.

2. $(x-y)^{\mathrm{T}}(\nabla f(x)-\nabla f(y))>0$.

The proposed algorithm is based on three key elements: the typical distance, the gradient criterion and the Double Box stopping rule. Their description is laid out in the following subsections.

\subsection{Typical distance}

A clustering procedure forms clusters of points by measuring the distance of a candidate point from the estimated centre of the cluster. This distance is checked against a threshold and a decision is made accordingly. The algorithm uses a typical distance defined by:

$$
r_{t} \equiv \frac{1}{M} \sum_{i=1}^{M}\left|x_{i}-L\left(x_{i}\right)\right|,
$$

where $x_{i}$ are starting-points for the local search procedure $L$, and $M$ is the number of the performed local searches. The main idea behind Eq. (1) is that after a number of iterations and a number of local searches the quantity $r_{t}$ will be a reasonable approximation for the mean radius of the regions of attraction. To see this note that if we denote by $M_{l}$ the number of times that the local search procedure discovered the minimizer $x_{l}^{*}$, then a basin radius may be defined as:

$$
R_{l}=\frac{1}{M_{l}} \sum_{j=1}^{M_{l}}\left|x_{l}^{(j)}-x_{l}^{*}\right|,
$$

where $\left\{x_{l}^{(j)}, j=1, \ldots, M_{l}\right\}=\left\{x_{i}, i=1, \ldots, M\right\} \cap A\left(x_{l}^{*}\right)$, i.e. $L\left(x_{l}^{(j)}\right)=x_{l}^{*}$. Since by definition $M=\sum_{l=1}^{w} M_{l}$, where $w$ is the number of local minima discovered so far, a mean basin radius may be defined as:

$$
\langle R\rangle \equiv \sum_{l=1}^{w} \frac{M_{l}}{M} R_{l}=\frac{1}{M} \sum_{l=1}^{w} \sum_{j=1}^{M_{l}}\left|x_{l}^{(j)}-x_{l}^{*}\right| .
$$

Comparing Eqs. (1), (2) and (3), it follows that $r_{t}=\langle R\rangle$.

\subsection{Gradient criterion}

The value of the objective function $f(x)$ at a point $x$ in the neighborhood of a local minimum $x^{*}$, can be estimated as:

$$
f(x) \simeq f\left(x^{*}\right)+\frac{1}{2}\left(x-x^{*}\right)^{\mathrm{T}} B^{*}\left(x-x^{*}\right),
$$

where $B^{*}$ is the Hessian matrix at the minimum $x^{*}$. By applying the gradient operator in each part of Eq. (4) we obtain:

$$
\nabla f(x) \simeq B^{*}\left(x-x^{*}\right) .
$$


In the same way for any other point $y$, in the neighborhood of a local minimum $x^{*}$, we have:

$$
\nabla f(y) \simeq B^{*}\left(y-x^{*}\right) .
$$

By subtracting (6) from (5) and by multiplying from the left with $(x-y)^{\mathrm{T}}$ we obtain:

$$
(x-y)^{\mathrm{T}}(\nabla f(x)-\nabla f(y)) \simeq(x-y)^{\mathrm{T}} B^{*}(x-y)>0
$$

(since $B^{*}$ is positive definite).

\subsection{Double Box stopping rule}

The most widely used stopping rule is that developed by Rinnooy Kan and Timmer [5], where the algorithm stops iterating if

$$
\frac{w(M-1)}{M-w-2}<w+\frac{1}{2} \text {. }
$$

$M$ being the number of total sample points (we consider uniform sampling) and $w$ the number of the located so far. The relation (8) may be rewritten as:

$$
M>2 w^{2}+3 w+2
$$

which means that in order to stop iterating the number of sample points must be greater than the square of the located local minima. This is undesirable for functions with many local minima and a more effective stopping rule must be employed. In the proposed method we use a termination rule that estimates the uncovered portion of the search space. A relative measure for this may be given by:

$$
U=1-\sum_{i=1}^{w} \frac{m\left(A_{i}\right)}{m(S)},
$$

where $w$ is the number of the discovered so far local minima and $m(S)$ is the Lebesgue measure of $S$. The quantity $m\left(A_{i}\right) / m(S)$ may be approximated by the fraction $T_{i} / T$, where $T_{i}$ is the sum of local searches that have ended up at the local minimum $x_{i}^{*}$, plus the sample points that have been allocated to the cluster centred at $x_{i}^{*}$, and $T$ is the total number of sample points. Hence an approximation for $U$ may be given by:

$$
U \simeq \tilde{U}=1-\sum_{i=1}^{w} \frac{T_{i}}{T} .
$$

Unfortunately, Eq. (11) always yields $\tilde{U}=0$ and hence the uncovered space cannot be estimated with the above relation. However, a larger box $S_{2}$, that contains $S$, is constructed in a way such that $m\left(S_{2}\right)=2 \times m(S)$. A unique (fake) local minimum is considered to be contained in $A_{0}=S_{2}-S$ with measure $m\left(A_{0}\right)=m(S)$. The uncovered portion of the search space is now given by:

$$
U=1-\frac{\sum_{i=0}^{w} m\left(A_{i}\right)}{m\left(S_{2}\right)}=1-\frac{m\left(A_{0}\right)}{m\left(S_{2}\right)}-\frac{\sum_{i=1}^{w} m\left(A_{i}\right)}{m\left(S_{2}\right)}=\frac{1}{2}-\frac{\sum_{i=1}^{w} m\left(A_{i}\right)}{m\left(S_{2}\right)} .
$$

The quantity $m\left(A_{i}\right) / m\left(S_{2}\right)$ is approximated again by the fraction $T_{i} / T, T$ being the total number of sample points in $S_{2}$ and hence:

$$
U \simeq \tilde{U}=\frac{1}{2}-\sum_{i=1}^{w} \frac{T_{i}}{T} .
$$

At every iteration we sample points from $S_{2}$ until we have collected $N$ points belonging to $S$. After $k$ iterations the total number of sample points $M_{k}$ and the quantity

$$
\delta_{k} \equiv \frac{k N}{M_{k}}
$$

has an expectation value (assuming that $\delta_{k}$ is i.i.d.)

$$
\langle\delta\rangle_{k}=\frac{1}{k} \sum_{i=1}^{k} \delta_{i}
$$

that asymptotically tends to $m(S) / m\left(S_{2}\right)=\frac{1}{2}$. The variance is given by

$$
\sigma_{k}^{2}(\delta)=\left\langle\delta^{2}\right\rangle_{k}-\langle\delta\rangle_{k}^{2}
$$

and tends to zero as $k \rightarrow \infty$. This is a smoother quantity than the expectation value and better suited for the following termination procedure: 
1. Initially set $a=0$ and $k=0$.

2. Sample from $S_{2}$ uniformly, until $N$ points fall in $S$.

3. Calculate the quantity $\delta_{k}=k N / M_{k}$.

4. Calculate the expectation value of $\delta_{k}$.

5. Calculate the deviation $\sigma(\delta)$.

6. Perform a step of the MinFinder algorithm (described in Section 2.1).

7. If one or more new minima are found, set $a=p \sigma^{2}(\delta)$ and repeat from step 2.

8. If $\sigma^{2}(\delta)<a$, TERMINATE, otherwise repeat from step 2 .

The parameter $p$ is in the range $(0,1)$. For small values of $p(p \rightarrow 0)$ the algorithm searches the area exhaustively, while for $p \rightarrow 1$ the algorithm terminates earlier, but perhaps prematurely. As a compromise between exhaustive and speed search, we suggest the value of $p=0.5$.

\section{Experimental results}

We compared the new method against Multistart (a description may be found in [10,11]) and Topographical Multilevel Single Linkage [6]. Other methods that one may consider, can be traced in $[13,14]$ for Simulated Annealing and in $[15,16]$ for Tabu Search. Simulated Annealing aims in discovering one global minimum only. The hybrid method [16] that combines Simulated Annealing, Tabu Search and a descent method is designed to discover all the global minima and some "important" local minima as well. We have used the Double Box stopping rule in all methods. All experiments have been repeated 50 times with different random number generator seed. The initial sample size was set to 20. In the following tables the columns "PROBLEM", "MINIMA", "FOUND", "FEVALS", "GEVALS" and "TIME" denote the name of the objective function, the known number of local minima, the average number of the discovered local minima, the average number of function evaluations performed, the average number of the gradient evaluations and the average CPU time. All the experiments were run on an AMD ATHLON 2400+ with 256 MB RAM running Slackware Linux v9.1. The local search procedure used in all methods was a BFGS implementation due to Powell [7].

In Tables 1, 2 and 3 we list the experimental results from the methods Multistart, TMLSL and MinFinder correspondingly. The results indicate that the proposed method is capable of finding almost all the minima of an objective function with less effort than other stochastic methods such as Multistart and TMLSL. The provided software has a very simple installation procedure and it can be easily installed in any UNIX operating system equipped with any ANSI C++ compiler.

\section{Software documentation}

\subsection{Distribution}

The package is distributed in a tar.gz file named MinFinder.tar.gz and under UNIX systems the user must issue the following commands to extract the associated files:

1. gunzip MinFinder.tar.gz.

2. $\operatorname{tar}$ xfv MinFinder.tar.

Table 1

Multistart results

\begin{tabular}{|c|c|c|c|c|c|}
\hline PROBLEM & MINIMA & FOUND & FEVALS & GEVALS & $\overline{\text { TIME }}$ \\
\hline CAMEL & 6 & 6 & 11138 & 10741 & 0.04 \\
\hline RASTRIGIN & 49 & 49 & 17714 & 16989 & 0.06 \\
\hline SHUBERT & 400 & 400 & 557668 & 535368 & 6.44 \\
\hline HANSEN & 527 & 527 & 586090 & 563131 & 8.71 \\
\hline $\operatorname{GKLS}(3,60)$ & 60 & 59 & 781378 & 691787 & 22.98 \\
\hline $\operatorname{GUILIN}(5,50)$ & 50 & 50 & 195392 & 190566 & 1.76 \\
\hline GOLDSTEIN & 4 & 4 & 15951 & 15570 & 0.08 \\
\hline SHEKEL5 & 5 & 5 & 25930 & 25431 & 0.75 \\
\hline SHEKEL7 & 7 & 7 & 28946 & 28382 & 1.11 \\
\hline SHEKEL10 & 10 & 10 & 30808 & 30175 & 1.09 \\
\hline HARTMAN3 & 3 & 3 & 11467 & 11086 & 0.14 \\
\hline HARTMAN6 & 2 & 2 & 16145 & 15764 & 0.52 \\
\hline
\end{tabular}


Table 2

TMLSL results

\begin{tabular}{|c|c|c|c|c|c|}
\hline PROBLEM & MINIMA & FOUND & FEVALS & GEVALS & TIME \\
\hline CAMEL & 6 & 6 & 3486 & 2643 & 0.03 \\
\hline RASTRIGIN & 49 & 49 & 23809 & 12195 & 0.78 \\
\hline SHUBERT & 400 & 400 & 337476 & 56597 & 127.47 \\
\hline GRIEWANK2 & 529 & 528 & 2233048 & 1840673 & 251.57 \\
\hline HANSEN & 527 & 527 & 838554 & 130642 & 451.38 \\
\hline $\operatorname{GKLS}(3,60)$ & 60 & 56 & 880641 & 357247 & 53.31 \\
\hline GUILIN(5, 50) & 50 & 50 & 220678 & 201519 & 3.21 \\
\hline GUILIN $(10,50)$ & 50 & 50 & 426882 & 401254 & 10.57 \\
\hline BRANIN & 3 & 3 & 1036 & 631 & 0.01 \\
\hline GOLDSTEIN & 4 & 4 & 4647 & 3871 & 0.04 \\
\hline SHEKEL5 & 5 & 5 & 7554 & 6366 & 0.23 \\
\hline SHEKEL7 & 7 & 6 & 11546 & 9936 & 0.43 \\
\hline SHEKEL10 & 10 & 9 & 18649 & 15786 & 0.65 \\
\hline HARTMAN3 & 3 & 3 & 1837 & 1559 & 0.03 \\
\hline HARTMAN6 & 2 & 2 & 860 & 621 & 0.03 \\
\hline
\end{tabular}

Table 3

MinFinder results

\begin{tabular}{|c|c|c|c|c|c|}
\hline PROBLEM & MINIMA & FOUND & FEVALS & GEVALS & TIME \\
\hline CAMEL & 6 & 6 & 1598 & 2187 & 0.02 \\
\hline RASTRIGIN & 49 & 49 & 1723 & 2975 & 0.08 \\
\hline SHUBERT & 400 & 400 & 17404 & 41849 & 7.07 \\
\hline GRIEWANK2 & 529 & 529 & 1035094 & 1190595 & 80.16 \\
\hline HANSEN & 527 & 527 & 60916 & 94382 & 15.79 \\
\hline $\operatorname{GKLS}(3,60)$ & 60 & 56 & 169280 & 297160 & 13.89 \\
\hline GUILIN $(5,50)$ & 50 & 50 & 84675 & 88111 & 1.21 \\
\hline GUILIN $(10,50)$ & 50 & 50 & 173186 & 179397 & 4.40 \\
\hline BRANIN & 3 & 3 & 498 & 604 & 0.01 \\
\hline GOLDSTEIN & 4 & 4 & 2197 & 2364 & 0.02 \\
\hline SHEKEL5 & 5 & 5 & 7144 & 7365 & 0.25 \\
\hline SHEKEL7 & 7 & 7 & 17125 & 17377 & 0.75 \\
\hline SHEKE110 & 10 & 10 & 21551 & 21661 & 0.90 \\
\hline HARTMAN3 & 3 & 3 & 1581 & 1737 & 0.03 \\
\hline HARTMAN6 & 2 & 2 & 1090 & 1194 & 0.05 \\
\hline
\end{tabular}

These steps create a directory named MinFinder with the following contents:

1. bin: A directory which is initially empty. After compilation of the package, it will contain the executable make_program.

2. examples: A directory that contains the test functions used in this article, written in ANSI C++ and the Fortran 77 version of the Six Hump Camel function.

3. include: A directory which contains the header files for all the classes of the package.

4. src: A directory containing the source files of the package.

5. Makefile: The input file to the make utility in order to build the tool. Usually, the user does not need to change this file.

6. Makefile.inc: The file that contains some configuration parameters, such as the name of the $\mathrm{C}++$ compiler, etc. The user must edit and change this file before installation.

\subsection{Installation}

The following steps are required in order to build the tool:

1. Uncompress the tool as described in the previous section.

2. cd Minfinder.

3. Edit the file Makefile. inc and change (if needed) the five configuration parameters.

4. Type make.

The five parameters in Makefile. inc are the following: 
1. CXX: It is the most important parameter. It specifies the name of the $\mathrm{C}++$ compiler. In most systems running the GNU C++ compiler this parameter must be set to $\mathrm{g}++$.

2. CC: If the user written programs are in $\mathrm{C}$, set this parameter to the name of the $\mathrm{C}$ compiler. Usually, for the GNU compiler suite, this parameter is set to gcc.

3. F77: If the user written programs are in Fortran 77, set this parameter to the name of the Fortran 77 compiler. For the GNU compiler suite a usual value for this parameter is $\mathrm{g} 77$.

4. F77FLAGS: The compiler GNU FORTRAN 77 (g77) appends an underscore to the name of all subroutines and functions after the compilation of a Fortran source file. In order to prevent this from happening we can pass some flags to the compiler. Normally, this parameter must be set to -fno-underscoring.

5. ROOTDIR: Is the location of the MinFinder directory. It is critical for the system that this parameter is set correctly. In most systems, it is the only parameter which must be changed.

\subsection{User written subprograms}

In Example 1 we see the template of the objective function in the $\mathrm{C}$ programming language. The same scheme is used also in $\mathrm{C}++$, but the code has the line

extern ' $\mathrm{C}$ ' \{

before the functions and the line

\}

after them, in order to prevent the compiler from generating symbols that will not cause problem to the linking process. The template for Fortran 77 is given in Example 2. The symbol d denotes the dimension of the objective function. The meaning of the functions are the following:

1. getdimension(): It returns the dimension of the objective function.

2. getleftmargin(left): It fills the double precision array left with the left margins of the objective function.

3. getrightmargin(right): It fills the double precision array right with the right margins of the objective function.

4. funmin(x): It returns the value of the objective function evaluated at point $x$.

5. $\operatorname{granal}(\mathrm{x}, \mathrm{g})$ : It returns in a double precision array $\mathrm{g}$ the gradient of the objective function at point $\mathrm{x}$.

\subsection{The utility make_program}

After the compilation of the package, the executable make_program will be placed in the subdirectory bin in the distribution directory. This program creates the final executable and it takes as its only argument the name of the file containing the objective function. The utility checks the suffix of the file and it uses the appropriate compiler. If this suffix is .cc or .c++ or .CC or .cpp, then it invokes the $\mathrm{C}++$ compiler. If the suffix is .f or .F or .for then it invokes the Fortran 77 compiler. Finally, if the suffix is .c it invokes the $\mathrm{C}$ compiler.

\subsection{The utility MinFinder}

After the compilation of the objective function with the tool make_program the executable MinFinder is created. This executable can take the following arguments in the command line:

1. -h: The program prints a help screen to the user and stops.

2. -s size: The integer parameter size is used as the size of the sample (i.e. $N=$ size). The default value for this parameter is 20 .

3. -o filename: The string parameter filename specifies a file where all the discovered local minima will be disposed after the termination of the program.

4. -p level: The integer parameter level can take only two values: 0 or 1 . If the value is 0 , then no output will be sent to the standard output. If the value is 1 , then after each iteration, the algorithm prints a line displaying the number of iterations, the number of located minima, the total number of function calls, the total number of gradient calls, the value of $\sigma^{2}(\delta)$ and the value of $a$ used in the Double Box stopping rule. The default value for this parameter is 0 .

5. -r seed: The integer parameter seed specifies the seed for the random number generator. It can assume any integer value. 
4.6. A working example

Consider the Six Hump Camel function given by

$$
f(x)=4 x_{1}^{2}-2.1 x_{1}^{4}+\frac{1}{3} x_{1}^{6}+x_{1} x_{2}-4 x_{2}^{2}+4 x_{2}^{4}, \quad x \in[-5,5]^{2}
$$

with 6 local minima. The implementation of this function in C++ and in Fortran 77 is shown in Examples 3 and 4. Let the file with the $\mathrm{C}++$ code be named camel. $\mathrm{CC}$ and that with the Fortran code Camel.f. Let these files be located in the examples subdirectory. Change to the examples subdirectory and create the MinF inder executable with the make_program command:

../bin/make_program camel.cc

or for the Fortran 77 version

../bin/make_program camel.f

The make_program responds:

RUN./MinFinder IN ORDER TO RUN THE PROBLEM

Run MinFinder by issuing the command:

./MinFinder -o camel.out -p 1 -r 7

The resulting output appears as:

\begin{tabular}{|c|c|c|c|c|c|c|c|c|c|}
\hline$r s=$ & 1 & $m=$ & 1 & $1 s=$ & 24 & & נת & delta= & stopat $=1.9763 e-32$ \\
\hline iters $=$ & 2 & minimum= & 2 & fevals $=$ & 89 & evals $=$ & 106 & delta $=$ & stopat $=$ \\
\hline ters $=$ & 3 & minimum= & 3 & fevals $=$ & 151 & gevals= & 178 & $\operatorname{delt} a=6.7063$ & stopat $=3.35$ \\
\hline ers $=$ & 4 & $\operatorname{mini}$ & 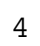 & fevals $=$ & 236 & $s=$ & 272 & & stop \\
\hline$r s=$ & $ᄃ$ & $\operatorname{mini}$ & 6 & fevals $=$ & 282 & $s=$ & 331 & 5 & to \\
\hline $\operatorname{ers}=$ & 6 & $\operatorname{mini}$ & 6 & fevals $=$ & 386 & $s=$ & 446 & 05 & stop \\
\hline $\operatorname{cers}=$ & & $\operatorname{mir}$ & 6 & fev & 520 & $S=$ & 5 & & $=1.7$ \\
\hline$r s=$ & & $u m=$ & 6 & fev & 04 & $s=$ & & 5 & 05 \\
\hline Eers $=$ & & $\mathrm{um}=$ & 6 & fevals $=$ & 680 & $\operatorname{vals}=$ & 77 & $a=2.2972 e-05$ & stopat $=1.7938 e-05$ \\
\hline & & $\mathrm{um}=$ & 6 & & 804 & & & $a=2.0464 e-05$ & stopat $=1.7938 e-05$ \\
\hline Eers $=$ & & ninimum= & & fevals $=$ & 888 & $\operatorname{vals}=$ & 13 & delta $=1.8794 e-05$ & stopat $=1.7938 e-05$ \\
\hline
\end{tabular}

All minima are discovered by iteration 5, however the program continued until iteration 11, because delta, which corresponds to the quantity $\sigma^{2}(\delta)$, at the 5 th iteration was not lower than stopat (the quantity $a$ in our algorithm). The discovered minima are written to the file camel. out, the contents of which are listed below:

2

$\begin{array}{ccc}-1.703606715 & 0.7960835687 & -0.2154638244 \\ 0.0898420131 & -0.712656403 & -1.031628453 \\ -0.0898420131 & 0.712656403 & -1.031628453 \\ -1.607104753 & -0.5686514549 & 2.10425031 \\ 1.703606715 & -0.7960835687 & -0.2154638244 \\ 1.607104753 & 0.5686514549 & 2.10425031\end{array}$

In the first line the single entry (number 2) denotes the dimensionality of the problem. In the second line the single entry (number 6) denotes the number of the discovered local minima. In each of the following lines there are three entries. The first two correspond to the parameter values of the minimizer, while the third to the corresponding value of the objective function.

\section{Appendix A. Test functions}

We list the test functions used in our experiments, the associated search domains and the number of the known local minima. These functions are standard test functions in the area of global optimization and further information about them can be found in [12] and at the URL:

http://www.imm.dtu.dk/ km/Globopt/testex/testproblems.html 


\section{Camel}

$$
f(x)=4 x_{1}^{2}-2.1 x_{1}^{4}+\frac{1}{3} x_{1}^{6}+x_{1} x_{2}-4 x_{2}^{2}+4 x_{2}^{4}, x \in[-5,5]^{2} \text { with } 6 \text { local minima. }
$$

\section{Rastrigin}

$$
f(x)=x_{1}^{2}+x_{2}^{2}-\cos \left(18 x_{1}\right)-\cos \left(18 x_{2}\right), x \in[-1,1]^{2} \text { with } 49 \text { local minima. }
$$

\section{Shubert}

$$
f(x)=-\sum_{i=1}^{2} \sum_{j=1}^{5} j\left\{\sin \left((j+1) x_{i}\right)+1\right\}, x \in[-10,10]^{2} \text { with } 400 \text { local minima. }
$$

\section{Griewank2}

$$
f(x)=1+\frac{1}{200} \sum_{i=1}^{2} x_{i}^{2}-\prod_{i=1}^{2} \frac{\cos \left(x_{i}\right)}{\sqrt{(i)}}, x \in[-100,100]^{2} \text { with } 529 \text { local minima. }
$$

\section{Hansen}

$$
f(x)=\sum_{i=1}^{5} i \cos \left[(i-1) x_{1}+i\right] \sum_{j=1}^{5} j \cos \left[(j+1) x_{2}+j\right], x \in[-10,10]^{2} \text { with } 527 \text { local minima. }
$$

\section{Gkls}

$f(x)=\operatorname{Gkls}(x, n, w)$, is a function with $w$ local minima, described in [9], $x \in[-1,1]^{n}, n \in[2,100]$. In our experiments we use $n=3$ and $w=60$.

\section{Guilin Hills}

$f(x)=3+\sum_{i=1}^{n} c_{i} \frac{x_{i}+9}{x_{i}+10} \sin \left(\frac{\pi}{1-x_{i}+1 / 2 k_{i}}\right), x \in[0,1]^{n}, c_{i}>0$ and $k_{i}$ are positive integers. This function has $\prod_{i=1}^{n} k_{i}$ local minima. In our experiments we use $n=5,10$ and we have arranged the values of $k_{i}$ so that the number of minima was 50. These cases are entitled as $\operatorname{GUILIN}(5,50)$ and $\operatorname{GUILIN}(10,50)$ in the following tables.

\section{Branin}

$f(x)=\left(x_{2}-\frac{5.1}{4 \pi^{2}} x_{1}^{2}+\frac{5}{\pi} x_{1}-6\right)^{2}+10\left(1-\frac{1}{8 \pi}\right) \cos \left(x_{1}\right)+10$ with $-5 \leqslant x_{1} \leqslant 10,0 \leqslant x_{2} \leqslant 15$. The function has 3 minima in the specified range.

\section{GoldStein \& Price}

$$
\begin{aligned}
f(x)= & {\left[1+\left(x_{1}+x_{2}+1\right)^{2}\left(19-14 x_{1}+3 x_{1}^{2}-14 x_{2}+6 x_{1} x_{2}+3 x_{2}^{2}\right)\right] } \\
& \times\left[30+\left(2 x_{1}-3 x_{2}\right)^{2}\left(18-32 x_{1}+12 x_{1}^{2}+48 x_{2}-36 x_{1} x_{2}+27 x_{2}^{2}\right)\right] .
\end{aligned}
$$

The function has 4 local minima in the range $[-2,2]^{2}$.

\section{Shekel 5}

$$
f(x)=-\sum_{i=1}^{5} \frac{1}{\left(x-a_{i}\right)\left(x-a_{i}\right)^{\mathrm{T}}+c_{i}}
$$

with $x \in[0,10]^{4}$ and

$$
a=\left(\begin{array}{llll}
4 & 4 & 4 & 4 \\
1 & 1 & 1 & 1 \\
8 & 8 & 8 & 8 \\
6 & 6 & 6 & 6 \\
3 & 7 & 3 & 7
\end{array}\right)
$$

and

$$
c=\left(\begin{array}{l}
0.1 \\
0.2 \\
0.2 \\
0.4 \\
0.4
\end{array}\right)
$$

The function has 5 local minima in the specified range.

\section{Shekel 7}

$$
f(x)=-\sum_{i=1}^{7} \frac{1}{\left(x-a_{i}\right)\left(x-a_{i}\right)^{\mathrm{T}}+c_{i}}
$$


with $x \in[0,10]^{4}$ and

$$
a=\left(\begin{array}{llll}
4 & 4 & 4 & 4 \\
1 & 1 & 1 & 1 \\
8 & 8 & 8 & 8 \\
6 & 6 & 6 & 6 \\
3 & 7 & 3 & 7 \\
2 & 9 & 2 & 9 \\
5 & 3 & 5 & 3
\end{array}\right)
$$

and

$$
c=\left(\begin{array}{l}
0.1 \\
0.2 \\
0.2 \\
0.4 \\
0.4 \\
0.6 \\
0.3
\end{array}\right) .
$$

The function has 7 local minima in the specified range.

\section{Shekel 10}

$$
f(x)=-\sum_{i=1}^{10} \frac{1}{\left(x-a_{i}\right)\left(x-a_{i}\right)^{\mathrm{T}}+c_{i}}
$$

with $x \in[0,10]^{4}$ and

$$
a=\left(\begin{array}{cccc}
4 & 4 & 4 & 4 \\
1 & 1 & 1 & 1 \\
8 & 8 & 8 & 8 \\
6 & 6 & 6 & 6 \\
3 & 7 & 3 & 7 \\
2 & 9 & 2 & 9 \\
5 & 5 & 3 & 3 \\
8 & 1 & 8 & 1 \\
6 & 2 & 6 & 2 \\
7 & 3.6 & 7 & 3.6
\end{array}\right)
$$

and

$$
c=\left(\begin{array}{l}
0.1 \\
0.2 \\
0.2 \\
0.4 \\
0.4 \\
0.6 \\
0.3 \\
0.7 \\
0.5 \\
0.6
\end{array}\right) .
$$

\section{Hartman 3}

$$
f(x)=-\sum_{i=1}^{4} c_{i} \exp \left(-\sum_{j=1}^{3} a_{i j}\left(x_{j}-p_{i j}\right)^{2}\right)
$$

with $x \in[0,1]^{3}$ and

$$
a=\left(\begin{array}{ccc}
3 & 10 & 30 \\
0.1 & 10 & 35 \\
3 & 10 & 30 \\
0.1 & 10 & 35
\end{array}\right)
$$


and

$$
c=\left(\begin{array}{c}
1 \\
1.2 \\
3 \\
3.2
\end{array}\right)
$$

and

$$
p=\left(\begin{array}{ccc}
0.3689 & 0.117 & 0.2673 \\
0.4699 & 0.4387 & 0.747 \\
0.1091 & 0.8732 & 0.5547 \\
0.03815 & 0.5743 & 0.8828
\end{array}\right)
$$

The function has 3 minima in the specified range.

\section{Hartman 6}

$$
f(x)=-\sum_{i=1}^{4} c_{i} \exp \left(-\sum_{j=1}^{6} a_{i j}\left(x_{j}-p_{i j}\right)^{2}\right)
$$

with $x \in[0,1]^{6}$ and

$$
a=\left(\begin{array}{cccccc}
10 & 3 & 17 & 3.5 & 1.7 & 8 \\
0.05 & 10 & 17 & 0.1 & 8 & 14 \\
3 & 3.5 & 1.7 & 10 & 17 & 8 \\
17 & 8 & 0.05 & 10 & 0.1 & 14
\end{array}\right)
$$

and

$$
c=\left(\begin{array}{c}
1 \\
1.2 \\
3 \\
3.2
\end{array}\right)
$$

and

$$
p=\left(\begin{array}{llllll}
0.1312 & 0.1696 & 0.5569 & 0.0124 & 0.8283 & 0.5886 \\
0.2329 & 0.4135 & 0.8307 & 0.3736 & 0.1004 & 0.9991 \\
0.2348 & 0.1451 & 0.3522 & 0.2883 & 0.3047 & 0.6650 \\
0.4047 & 0.8828 & 0.8732 & 0.5743 & 0.1091 & 0.0381
\end{array}\right) .
$$

The function has 2 local minima in the specified range.

\section{Appendix B. Examples}

Example 1 (Formulation in C).

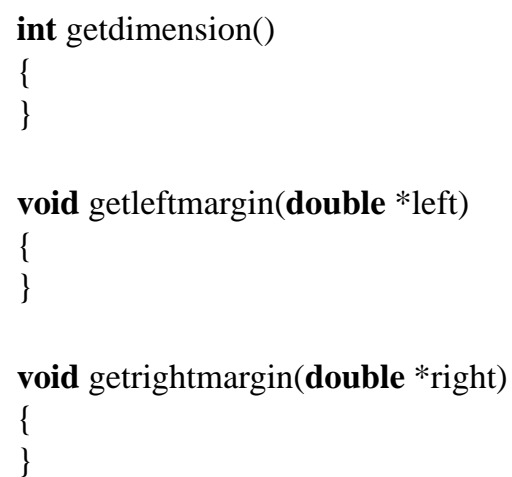


void granal $($ double $* x$,double $* g)$

\{

\}

Example 2 (Formulation in Fortran 77).

integer function getdimension()

getdimension $=\mathrm{d}$

end

subroutine getleftmargin(left)

double precision left $(\mathrm{d})$

end

subroutine getrightmargin(right)

double precision right(d)

end

double precision function funmin $(\mathrm{x})$

double precision $\mathrm{x}(\mathrm{d})$

end

subroutine granal $(\mathrm{x}, \mathrm{g})$

double precision $\mathrm{x}(\mathrm{d})$

double precision $\mathrm{g}(\mathrm{d})$

end

Example 3 (Implementation of Camel function in $\mathrm{C}++$ ).

\section{extern "C" $\mathrm{C}$}

int getdimension()

\{

return 2;

\}

void getleftmargin(double *left)

\{

left $[0]=-5.0$;

left $[1]=-5.0$

\}

void getrightmargin $($ double $*$ right $)$

\{

$\operatorname{right}[0]=5.0$;

right $[1]=5.0$

\}

double funmin (double *x)

\{

double $\mathrm{x} 1=\mathrm{x}[0], \mathrm{x} 2=\mathrm{x}[1]$;

return $4 * \mathrm{x} 1 * \mathrm{x} 1-2.1 * \mathrm{x} 1 * \mathrm{x} 1 * \mathrm{x} 1 * \mathrm{x} 1+$

$\mathrm{x} 1 * \mathrm{x} 1 * \mathrm{x} 1 * \mathrm{x} 1 * \mathrm{x} 1 * \mathrm{x} 1 / 3.0+\mathrm{x} 1 * \mathrm{x} 2-4 * \mathrm{x} 2 * \mathrm{x} 2+4 * \mathrm{x} 2 * \mathrm{x} 2 * \mathrm{x} 2 * \mathrm{x} 2$;

\} 


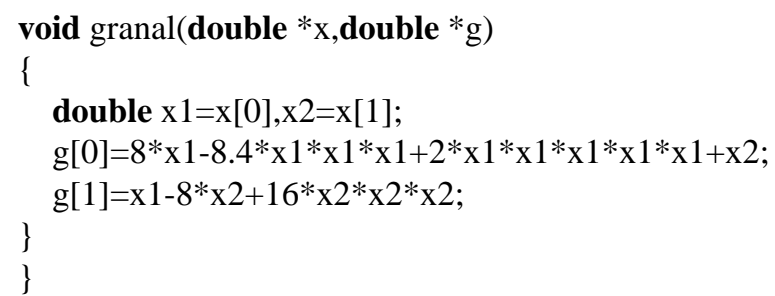

Example 4 (Implementation of Camel function in Fortran 77).

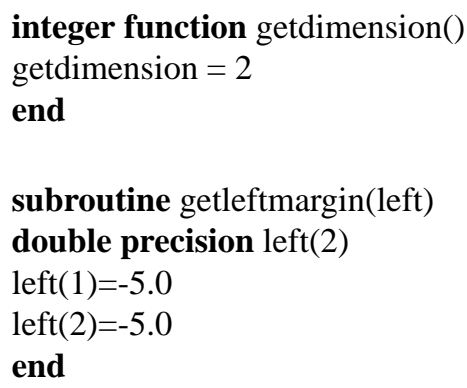

\section{References}

[1] R.W. Becker, G.V. Lago, A global optimization algorithm, in: Proc. 8th Allerton Conference on Circuits and Systems Theory, 1970.

[2] A.A. Törn, A search clustering approach to global optimization, in: L.C.W. Dixon, G.P. Szegö (Eds.), Towards Global Optimizations, vol. 2, North-Holland, Amsterdam, 1978.

[3] C.G.E. Boender, A.H.G. Rinnooy Kan, G.T. Timmer, L. Stougie, A stochastic method for global optimization, Math. Programm. 22 (1982) 125-140.

[4] A.H.G. Rinnooy Kan, G.T. Timmer, Stochastic global optimization methods, Part I: Clustering methods, Math. Programm. 39 (1987) $27-56$.

[5] A.H.G. Rinnooy Kan, G.T. Timmer, Stochastic global optimization methods, Part II: Multilevel methods, Math. Programm. 39 (1987) 57-78.

[6] M.M. Ali, C. Storey, Topographical multilevel single linkage, J. Global Optimization 5 (1994) 349-358.

[7] M.J.D. Powel, A tolerant algorithm for linearly constrained optimization calculations, Math. Programm. 45 (1989) 547.

[8] R. Fletcher, A new approach to variable metric algorithms, Comput. J. 13 (1970) 317-322.

[9] M. Gaviano, D.E. Ksasov, D. Lera, Y.D. Sergeyev, Software for generation of classes of test functions with known local and global minima for global optimization, ACM Trans. Math. Software 29 (2003) 469-480.

[10] A. Törn, A. Žilinskas, Global Optimization, Lecture Notes in Comput. Sci., vol. 350, Springer, Heidelberg, 1987. 
[11] L.C.W. Dixon, G.P. Szegö, The global optimization: An introduction, in: L.C.W. Dixon, G.P. Szegö (Eds.), Towards Global Optimization, vol. 2, North-Holland, Amsterdam, 1978, pp. 1-15

[12] C.A. Floudas, P.M. Pardalos, C. Adjiman, W. Esposoto, Z. Gümüs, S. Harding, J. Klepeis, C. Meyer, C. Schweiger, Handbook of Test Problems in Local and Global Optimization, Kluwer Academic Publishers, Dordrecht, 1999.

[13] S. Kirkpatrick, C.D. Gelatt, M.P. Vecchi, Optimization by simulated annealing, Science 220 (4) (1983) 671-680.

[14] A. Corana, M. Marchesi, C. Martini, S. Ridella, Minimizing multimodal functions of continuous variables with the "simulated annealing" algorithm, ACM Trans. Math. Software 13 (1987) 262-280.

[15] R. Chelouah, P. Siarry, Tabu search applied to global optimization, European J. Oper. Res. 123 (2000) 256-270.

[16] S. Salhi, N.M. Queen, A hybrid algorithm for identifying global and local minima when optimizing functions with many minima, European J. Oper. Res. 155 (2004) 51-67. 